Tissue velocity imaging of all patients and controls were accepted in apical two, four and long axis chamber views. Strain rate (SR) imaging was performed on all cases, peak atrial systolic left atrial SR (LASRa) were measured at each segment (septal, lateral, posterior, anterior, and inferior walls of left atrium), mean LASRa was calculated by averaging data in all segments.

Results Compared with controls, LAFS and mLASRs of HCM and HLVH were significantly higher $(p<0.05)$. Moreover, LAFS and mLASRs of HCM were significantly higher than HLVH $(p<0.05)$. mLASRa correlated positively with LAFS of HCM and HLVH respectively $(\mathrm{r}=0.81,0.88 ; \mathrm{p}<0.01,0.01)$.

Conclusions The change of left atrial function of HCM and HLVH presented as pump function increased, especially of HCM, which may take some clinical information to identify these two diseases.

\section{e0687 THE APPLICATION OF VECTOR FLOW MAPPING IN QUANTITATIVE ASSESSMENT OF REGIONAL MYOCARDIAL FUNCTION IN CAD PATIENTS}

doi:10.1136/hrt.2010.208967.687

Xu Mingjun, Zhang Mei, Zhang Yun. Cardiovascular Department, Qilu Hosptial, Shandong University

Background Until now, the study of regional infarct and ischaemic myocardial function in patients with coronary atherosclerotic heart disease is still mainly focused on the abnormal wall motion, how the blood flow dynamics affected by the regional abnormal wall motion in $\mathrm{CAD}$ patients is remains unknown. The purposes of the present study are to investigate the blood flow dynamics adjacent to the regional ischaemic and infarct myocardial segments, to explore the correlation between the blood flow dynamics and wall motion and to explore sensitive indexes showing blood flow dynamics change caused by abnormal wall motion in CAD patients.

Methods 43 CAD patients were enrolled in our study. Velocity Vector Imaging technique was used to evaluate the wall motion so as to classify the myocardial segments as normal, ischaemic and infarct by systolic peak strain. Vector Flow Mapping (VFM) technique was performed to show the blood flow dynamics in the heart chamber. The blood flow volume heading towards or against the apex in different periods of a heart beat and the time to every peak flow were measured. Results Compared to normal group, systole $Q+$ is higher $(p<0.01)$, diastole $Q+$ is lower $(p<0.01)$, T2 is shorter $(p<0.01)$ in ischaemic group. Compared to non-infarct group, systole $Q+$ is higher $(p<0.05)$, diastole $Q+$ is lower $(p<0.01)$, T2 is shorter $(p<0.05)$ in infarct group. There are correlations between indexes derived by VFM and Velocity Vector Imaging technique. Systole $\mathrm{Q}+$ is a better index, when the cutoff value reaches 25.32 and 28.046; it has a high sensitivity and specificity for differentiating ischaemic and infarct segments.

Conclusion VFM is a novel noninvasive tool to assess the blood flow dynamics and can be used to evaluate the cardiac function.

\section{e0688 INTRAVASCULAR ULTRASOUND CRITERIA FOR THE ASSESSMENT OF THE FUNCTIONAL SIGNIFICANCE OF INTERMEDIATE CORONARY ARTERY STENOSIS}

doi:10.1136/hrt.2010.208967.688

Cheng Xunming, He Guoxiang, Liu Jianping, Tong Shifei. Department of Cardiology, Southwest Hospital, Third Military Medical University \& Chongqing Institute of Interventional Cardiology

Introduction In recent years, intravascular ultrasound (IVUS) has evolved as a valuable adjunct to angiography. IVUS allows precise tomographic measurement of lumen area and plaque size, distribution and, to some extent, composition. It is essential in clinic deci- sion making. Myocardial fractional flow reserve $\left(\mathrm{FFR}_{\text {myo }}\right)$ is a special index of the functional severity of coronary stenosis. Although the application of $\mathrm{FFR}_{\text {myo }}$ to assess intermediate coronary lesion is widely performed in some centers in developed countries, its use in China was lagged. Because it is relatively expensive to measure $\mathrm{FFR}_{\text {myo, }}$ it will be beneficial to save the expenses and to short operation time if CAG and IVUS criteria could be clinically used as tools to discriminate functional significant of intermediate stenosis. Materials and methods In 46 lesions of intermediate severity (eg, $40 \%$ to $60 \%$ diameter stenosis) we assessed 1 . by pressure wire: myocardial fractional flow reserve $\left(\mathrm{FFR}_{\mathrm{myo}}\right.$, index of functional significance), and 2 . by IVUS: minimal lumen cross-sectional area (MLA) and percent area stenosis at the lesion site. Receiver operating characteristic curve analysis was performed to establish the best cut-off values of IVUS indexes (ie, MLA and percent area stenosis) that were most predictive of $\mathrm{FFR}_{\text {myo }}<0.75$.

Results FFRmyo in 46 lesions of angiographic intermediate stenosis $(49 \pm 11 \%)$ was significantly lower than it was in angiographic normal artery $(0.83 \pm 0.15$ vs $0.97 \pm 0.02, p<0.01) .14$ lesions $(30 \%)$ were functionally critical (eg, FFR myo $<0.75$ ). By regression analysis, percent area stenosis had a significant inverse correlation with $\mathrm{FFR}_{\text {myo }}(\mathrm{r}=-0.68, \mathrm{p}<0.01)$. MLA showed a significant positive relation with $\mathrm{FFR}_{\text {myo }}(\mathrm{r}=0.63, \mathrm{p}<0.01)$. By receiver operating characteristic analysis, we identified a IVUS area stenosis $\geq 65 \%$ (sensitivity $100 \%$, specificity $72 \%$ ), a minimal lumen cross-sectional area $\leq 4 \mathrm{~mm}^{2}$ (sensitivity $93 \%$, specificity $77 \%$ ) to be the best cut-off values to fit with $\mathrm{FFR}_{\text {myo }}<0.75$.

Conclusion IVUS area stenosis $\geq 65 \%$ and minimal lumen crosssectional area $\leq 4 \mathrm{~mm}^{2}$ reliably identified functionally critical intermediate coronary stenosis.

\section{E0689 ECHOCARDIOGRAPHIC CHARACTERISTICS OF LEFT VENTRICULAR NONCOMPACTION WITH PAPILLARY MUSCLES INVOLVEMENT}

doi:10.1136/hrt.2010.208967.689

Jiang Lan, Xie Mingxing, Wang Xinfang, Yuan Li, Lu Xiaofang, Li Lin. Department of Ultrasonography, Tongji Medical College Huazhong University of Science and Technology

Objective To assess the ultrasonic characteristics of the left ventricular noncompaction with papillary muscles involvement.

Methods We reviewed the echocardiographic findings in eight patients having left ventricular noncompaction with papillary muscles involvement at our hospital. We performed transthoracic echocardiography including standard parasternal (short and long axis), apical (two-chamber, three-chamber and four-chamber), and nonstandard parasternal views for all these patients. The left ventricular segments involved in noncompaction, the mitral papilary muscles and the mitral valve were the key observation. When the transthoracic echocardiography of four patients were not satisfactory, contrast echocardiography was used.

Results In all 8 patients (5 of them with severe mitral regurgitation, two with hypertrophic cardiomyopathy), the myocardium of the left ventricular segments involved in noncompaction were consist of two layers: a thin, compacted epicardial and an extremely thickened endocardial layer with prominent trabeculations and deep recesses, mitral papillary muscles involved in whole or in part, the myocardium of the papillary muscles looked like moth-eaten, appearance of blood flow from the ventricular cavity into the intertrabecular recesses both in the noncompaction myocardium and papillary muscles as visualised by colour Doppler imaging. With contrast enhancement in four of eight patients, the left ventricular endocardial borders were clearly outlined, and contrast microbubbles filled into the intertrabecular recesses both in the noncompaction myocardium and papillary muscles. 